\title{
COMPARAÇÃO ENTRE COLA BIOLÓGICA E SUTURA EM CICATRIZAÇÃO DA PELE
}

COMPARISON BETWEEN BIOLOGIC GLUE AND SUTURE ON SKIN HEALING

\author{
Andy Petroianu, TCBC-MG ${ }^{1}$ \\ Arnaldo Alves Silva ${ }^{2}$ \\ Marco Antônio Barreto de Melo ${ }^{3}$ \\ Leonardo de Souza Vasconcellos ${ }^{4}$
}

\begin{abstract}
RESUMO: Objetivo: A utilização da cola biológica em diversos campos da cirurgia continua controversa, apesar do grande número de pesquisas. O objetivo do presente trabalho foi comparar a cicatrização da pele de ratos submetidos à sutura e à aplicação de cola biológica em distintos períodos pós-operatórios. Método: Em 10 ratos machos, foram confeccionados dois retalhos de pele em forma de "U" na região dorsal. Um dos retalhos foi suturado com fio de polipropileno 6-0, enquanto o outro foi fixado com cola biológica à base de gelatina-resorcinol formaldeído (GRF). Metade dos animais $(n=5)$ foi acompanhada por um período de oito dias, enquanto os outros cinco foram estudados durante 21 dias. Avaliaram-se os aspectos macro e microscópio das cicatrizes. Resultados: Após oito dias, a ferida em presença da cola biológica mostrou maior inflamação e reação cicatricial mais exuberante. Contudo, decorridos 21 dias, não houve diferença entre a cicatrização dos retalhos cutâneos em presença de fio de polipropileno ou cola biológica. Conclusão: Os resultados indicam que a cola biológica parece ser uma boa alternativa para a síntese de pele em rato.
\end{abstract}

Descritores: Sutura; Cola biológica; Cicatrização da pele.

\section{INTRODUÇÃO}

O avanço tecnológico tem permitido o desenvolvimento de materiais de síntese biodegradáveis e mais compatíveis com os tecidos vivos. As novidades mais marcantes foram o uso de grampeadores, do laser e das colas biológicas ${ }^{1}$. Apesar de propostas feitas por Young et al. ${ }^{2}$, pioneiros na utilização do coágulo de fibrina para promover aderências, somente a partir da década de 60 surgiram estudos mais aprofundados sobre as colas biológicas, do ponto de vista experimental e clínico.

Essas colas, também conhecidas pelo nome de adesivos tissulares, são substâncias capazes de estabelecer uma ligação entre sua estrutura molecular e a do tecido sobre o qual são aplicadas. Em geral, apresentam-se sob a forma de gel e sua conseqüente solidificação garante a aproximação e fixação das superfícies justapostas. Esse processo de solidificação varia de acordo com a estrutura molecular do adesivo, o meio sobre o qual é aplicado e fatores ambientais, como calor, luz e $\mathrm{pH}^{3,4}$.

Segundo Cooper et al. ${ }^{5}$, o método mais usado para acelerar a solidificação do adesivo é a mistura ao gel de uma substância polimerizante, que o torna mais eficiente quando aplicado sobre superfície seca e limpa, apesar de alguns adesivos serem compatíveis com certo grau de umidade $^{3}$. O adesivo ideal deve ser capaz de produzir uma união tissular estável, ser impermeável e reagir com o tecido vivo, solidificando rapidamente. Além disso, precisa ter consis-

1. Professor Titular do Departamento de Cirurgia da Faculdade de Medicina da UFMG, Docente-Livre da Faculdade de Medicina de Ribeirão Preto - USP. Docente-Livre da Escola Paulista de Medicina - UNIFESP. Doutor em Fisiologia e Farmacologia, Pesquisador IA do CNPq.

2. Nefrologista.

3. Mestre em Medicina pelo curso de Pós-graduação em Ginecologia e Obstetrícia da UFMG.

4. Acadêmico de Medicina da Faculdade Ciências Médicas de Minas Gerais. Bolsista de Iniciação Científica da FAPEMIG.

Recebido em 31/10/2000

Aceito para publicação em 6/3/2001

Trabalho realizado no Departamento de Cirurgia da Faculdade de Medicina da UFMG, com auxílio do CNPq e da FAPEMIG. 
tência elástica, não alterando as características do tecido sobre o qual é aplicado. A sua conservação, esterilização e manuseio têm que ser fáceis, além de ser desprovido de efeito tóxico, carcinogênico ou alergênico ${ }^{3,5}$. Por outro lado, a desinformação e a existência dos tabus em torno da cola biológica são responsáveis pela sua pouca utilização rotineira e alternativa no meio cirúrgico.

Vários autores obtiveram experimentalmente hemostasia adequada em lesões de fígado, rins e pele com o uso de colas biológicas ${ }^{3,6,7}$. Outros, observaram que a cola biológica confere maior resistência cicatricial em anastomoses intestinais com relação às anastomoses apenas com a sutura tradicional $1^{8,9,10}$.

Tidrick et al. ${ }^{11}$ adicionaram ao plasma a trombina purificada formando um coágulo capaz de fixar de maneira eficiente enxertos cutâneos e abreviar a cicatrização. Os estudos de Schlag et al. ${ }^{12}$ mostraram ausência de infecções nos retalhos cutâneos, provavelmente por ação anti-séptica da cola biológica. Esse resultado seria decorrente da capacidade da fibronectina, presente na cola biológica, de fixar debris e bactérias, facilitando sua opsopnização e fagocitose.

Frente à necessidade de mais estudos sobre os efeitos das colas biológicas na cicatrização da pele, o presente trabalho, pertencente a uma linha de pesquisas sobre cicatrização ${ }^{13-19}$, teve como objetivo a comparação da eficácia da cicatrização de retalhos cutâneos em ratos submetidos a sutura e cola biológica, em distintos períodos pós-operatórios.

\section{MÉTODO}

O presente trabalho foi realizado de acordo com as recomendações das Declarações de Helsinque e as Normas Internacionais de Proteção aos Animais, e aprovado por Comitê de Ética da Universidade Federal de Minas Gerais ${ }^{20,21}$.

Foram utilizados dez ratos machos, da raça Holtzman, pesando entre 200 e 250 gramas.

Sob anestesia inalatória com éter, em todos animais foram realizadas duas incisões em " $U$ " na pele da região dorsal, separando completamente o retalho de pele da fáscia muscular. Um dos retalhos foi cranial e o outro caudal, medindo 3,0 x 2,0cm cada um e poupando a musculatura. Em seguida, um dos retalhos de pele foi suturado com pontos separados simples, englobando toda a pele, utilizando-se fio de polipropileno monofilamentar 6-0. O outro retalho foi fixado com cola biológica à base de gelatina-resorcinol formaldeído (GRF). A cola e a sutura foram empregadas alternadamente nos dois retalhos. Assim, em metade dos animais o retalho cranial foi suturado e o caudal colado, enquanto na outra metade a síntese foi feita ao contrário.

Para a aplicação da cola, inicialmente, os ângulos livres do retalho foram fixados com dois pontos de polipropileno 6-0. Em seguida, a cola foi aplicada de forma contínua nas três bordas da incisão, penetrando na ferida operatória. Após deixar-se a ferida cirúrgica em repouso por três minutos, os dois pontos de polipropileno foram retirados (Figura 1).

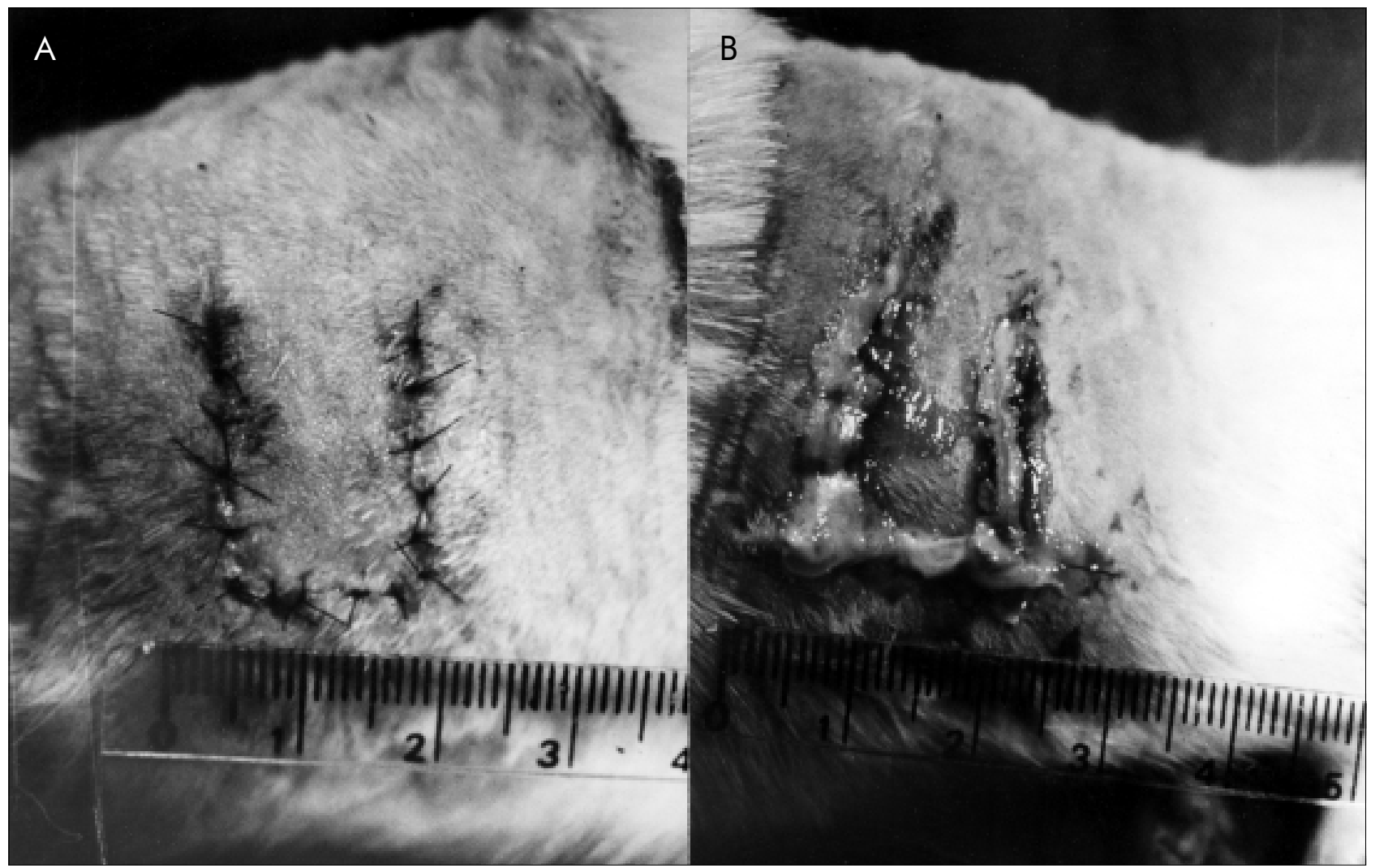

Figura 1 - Visão macroscópica de retalhos cutâneos fixados no pós-operatório imediato em rato. (a) Sutura (b) Cola biológica. 
O tronco do rato foi coberto por malha fina sobre a qual foi colocado um colete confeccionado a partir de frasco de plástico, utilizado para guardar soluções para infusão venosa. Esse colete teve como finalidade proteger a ferida operatória (Figura 2).

No pós-operatório, os retalhos foram avaliados diariamente. Os animais receberam água e ração para ratos, à vontade.

Os ratos foram divididos aleatoriamente em dois grupos $(n=5)$ de acordo com o tempo de acompanhamento, oito ou 21 dias, respectivamente. Decorridos esses períodos, os animais foram mortos com superdose inalatória de éter.

Estudou-se comparativamente a cicatrização com a cola biológica e com a sutura. O exame anatomopatológico foi realizado em preparações coradas por hematoxilina-eosina e tricrômico de Gomori, medindo-se a espessura da neoformação fibrosa cicatricial, nas três bordas do retalho cutâneo.

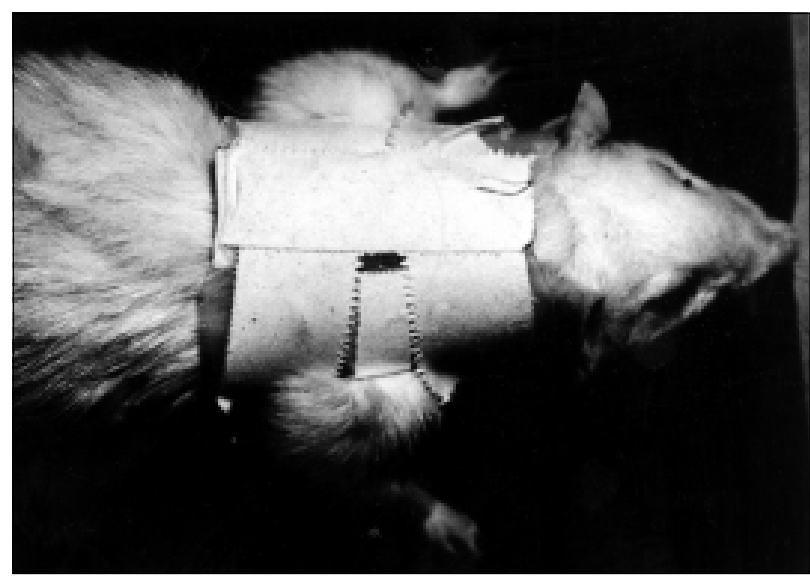

Figura 2 - Proteção das feridas operatórias do rato com colete confeccionado a partir de frasco de plástico.

\section{RESULTADOS}

Todos os ratos sobreviveram ao experimento e evoluíram satisfatoriamente. No oitavo dia pós-operatório, os retalhos apresentaram cicatriz mais frouxa. O retalho fixado com cola biológica desenvolveu maior reação inflamatória do que o suturado. Histologicamente, os retalhos fixados pela cola biológica tiveram padrão cicatricial mais heterogêneo, com reação inflamatória mais intensa e menor número de fibroblastos e feixes de colágeno (Figura 4b). Nos retalhos suturados, observou-se aumento discreto do colágeno e infiltrado inflamatório de polimorfonucleares, plasmócitos, linfócitos e macrófagos, além de congestão vascular.

No $21^{\circ}$ dia pós-operatório, todos os retalhos cutâneos tiveram cicatrização firme e sem áreas de infecção (Figura 3). Não houve diferença quanto ao aspecto morfológico, que foi mais homogêneo, com melhor arranjo de fibroblastos e fibras colágenas mais espessas, além de sua distribuição ser mais ordenada, em traves paralelas entre si, em relação ao aspecto do oitavo dia pós-operatório (Figura 4a). Na quantificação histométrica da espessura das bordas dos retalhos cutâneos, não houve diferença.

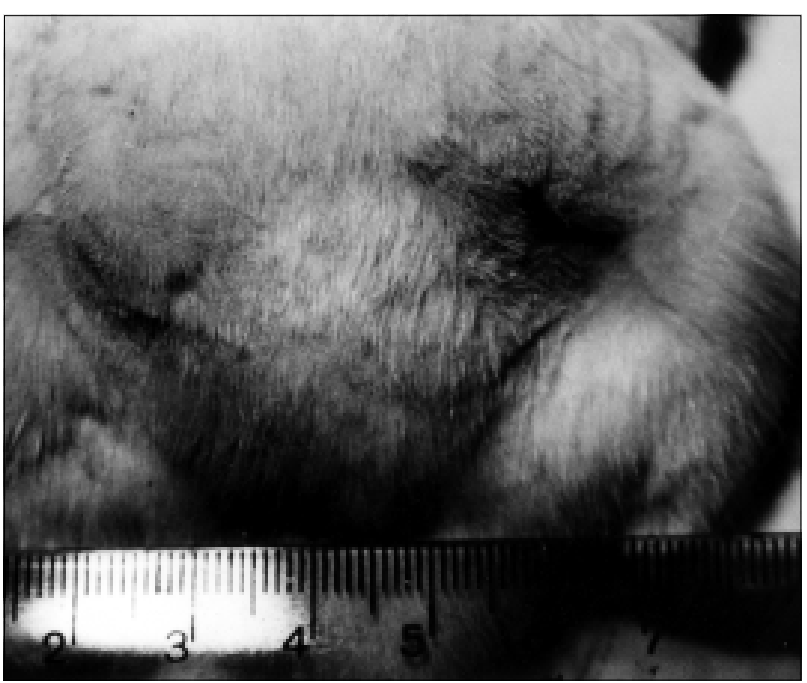

Figura 3 - Visão macroscópica de retalhos cutâneos fixados no $21^{\circ}$ dia pós-operatório. Cicatrização por sutura (à esquerda), já com os pontos retirados, e por cola biológica (à direita).

\section{DISCUSSÃO}

Fatores que afetam a cicatrização vêm sendo pesquisados continuamente. Embora existam diversos estudos experimentais sobre a relação entre a cicatrização e a cola biológica, a multiplicidade metodológica dificulta sua avaliação $0^{2,3,4,8,9,12}$.

Existem dois tipos de adesivos biológicos: os sintéticos e os naturais. Os adesivos naturais constituem o plasma, o crioprecipitado e a fibrina, que são totalmente degradados, aparentemente não tóxicos e pouco flogógenos. Já os adesivos sintéticos, são representados pela associação de gelatina, resorcinol e formaldeído (GRF). Estes, apesar de sintéticos, apresentam várias características peculiares aos adesivos naturais sendo considerados biológi$\cos ^{12}$. No presente trabalho, a cola biológica do tipo GRF foi usada por ser bem assimilada pelo organismo e não necessitar de superfície totalmente seca para ser aplicada ${ }^{3,5}$.

Os dados do presente trabalho indicam que, apesar de a cola demorar mais para conferir uma resistência adequada à cicatriz e também provocar uma inflamação maior na primeira semana, após 21 dias, a cicatrização da pele não foi diferente com os dois métodos. $\mathrm{O}$ resultado pôde ser comprovado tanto macro quanto microscopicamente. $\mathrm{Na}$ terceira semana todas as feridas encontravam-se no mesmo estádio cicatricial, ou seja, na fase de maturação, mostrando tecido conectivo bem formado e rico em fibras colágenas ordenadas.

É interessante destacar que nenhum animal desenvolveu infecção de ferida operatória. Atribuímos esse fato à resistência natural dos ratos aliada aos cuidados de anti-sepsia 


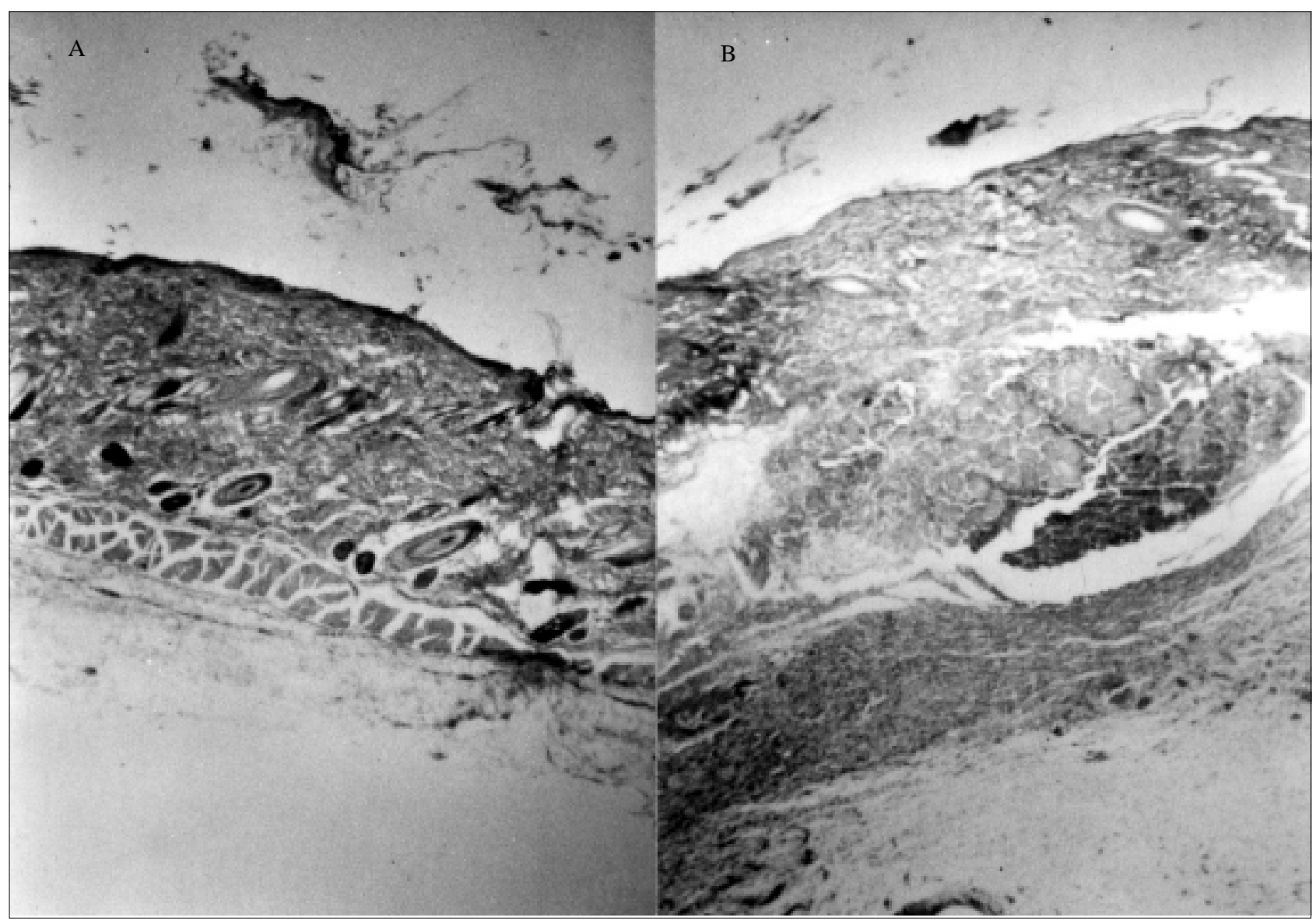

Figura 4 - Avaliação histológica dos retalhos cutâneos no 21o dia pós-operatório. Coloração H.E. (a) Sutura (b) Cola biológica.

operatória e aos curativos pós-operatórios diários. A provável ação anti-séptica da cola biológica pode ter contribuído para manter a ferida limpa, mas é importante ressaltar que os retalhos suturados também não tiveram infecção.
Concluindo, de acordo com os resultados do presente trabalho em ratos, a cola biológica parece ser, a médio prazo, uma alternativa para a síntese de feridas cutâneas tão boa quanto a sutura.

\begin{abstract}
Background: The use of biological glue in surgery continues to be controversial in spite of the great number of investigations. The present work compared the rat skin graft healing submitted to suture and biological glue in different post-operative periods. Methods: Two grafts in " $U$ " were performed on the dorsal area of 10 male rats. One graft was sutured with 6-0 polipropilen thread, while the other was put together with biological glue of gelatin-resorcinol formaldeid $(G R F)$. Half of the animals $(n=5)$ were followed during an eight days period, and the other five were studied during 21 days. The macro and microscopic aspects of the skin healing were assessed. Results: After eight days, the wound in presence of biologic glue showed more intense inflammation and an exuberant healing reaction. However, after 21 days, there was no difference between the cutaneous healing with 6-0 polipropilen and GRF glue. Conclusion: In the present study the biological glue was as good alternative for skin synthesis in rat as suture with polipropilen.
\end{abstract}

Key Words: Suture; Biologic glue; Skin cicatrization. 


\section{REFERÊNCIAS}

1. Hardy TG, Pace WG, Maney JW et al. Biofragmentable ring for sutureless bowel anastomosis. Dis Colon Rectum, 1985, 28: 484-490.

2. Young JZ, Medawar PB. Fibrin suture of peripheral nerves. Lancet, 1940, 239: 126-128.

3. Tatooles CJ, Braunwald NS. The use of crosslinked gelatin as a tissue adhesive to control hemmorrhage from liver and kidney. Surgery, 1966, 60: 857-861.

4. Koehnlein HE, Lemperle G. Experimental studies with a new gelatin-resorcin-formaldehyde glue. Surgery, 1969, 66: 377-382.

5. Cooper CN, Falb RD. Surgical adhesives. Ann NY Acad Sci, 1968, 146: 214-224.

6. Weisgerber G, Douvin D, Huguet C. La biologique GRF appliquée aux hépatectomies chez le rat. Ann Chir, 1974, 108: 485-500.

7. Souza C, Hartung TN, Colares C et al. Uso do Colagel ${ }^{\circledR}$ no trauma hepático, estudo experimental em ratos. Acta Cir Bras, 1989, 4(Supl 1): 87.

8. Scheele J, Herzog J, Mühe E. Fibrin glue protection of digestive anastomosis. Zentralbl Chir, 1978, 103: 13251336.

9. Ascherl R, Scherer M, Stemberger A et al. Renforcement des anastomosis coliques a l'aide de colle de fibrine. In: Scheele J ed. Finbrinklebung. Berlin, Springer Verlag, 1984, p 120-126.

10. Giordano L, Massa G, Vitale M. Fibrin glue in gastroenteric surgery. Minerva Chir, 1989, 44: 1371-1374.

11. Tidrick RT, Warner ED. Fibrin fixation of skin transplants. Surgery, 1944, 15: 90-95.

12. Schlag G, Redl H, Turnher M et al. The importance of Fibrin in Wound Repair. In: Schlag G, Edhl H, eds. General surgery and abdominal surgery. Berlin, Springer-Verlag, 1986, 6: 3-12.

13. Arantes VN, Silva AA, Petroianu A et al. Efeito da metilprednisolona sobre a tensão anastomótica jejunal. Arq Gastroenterol, 1994, 31: 97-101.

14. Melo MAB, Almeida LM, Petroianu A, et al. Cicatrização de anastomose colônica em ratos submetidos a diferentes preparos colônicos. Rev Bras Colo-Proct, 1996; 16: 19-22.

15. Arantes VA, Okawa RY, Petroianu A, et al. Influência da icterícia obstrutiva na cicatrização de pele e de anastomose jejunal em ratos. Rev Col Bras Cir, 1999, 26: 269-273.
16. Petroianu A, Silva RTF, Parreira LM et al. Alterações morfológicas do fígado após secção hepática parcial e omentoplastia. Rev Col Bras Cir, 1998, 25: 15-17.

17. Money SR, Petroianu A, Stillman R. Influence of type of suture material on the patency of arteriovenous anastomosis. Rev Med Minas Gerais, 1998, 8: 146-147.

18. Petroianu A, Trapiello VN, Esquerdo CRM et al. Efeito da secção hepática parcial e omentoplastia na regeneração hepática de cão. Rev Col Bras Cir, 1999, 26: 181184.

19. Gonçalves RM, Esquerdo CRM, Petroianu A et al. Influência de aderências peritoneais e fio cirúrgico na tensão de ruptura da parede abdominal em ratos. Rev Col Bras Cir, 2000, 27: 147-152.

20. Cooper JE. Ethics and laboratory animals. Vet Rec, 1985, 116: 594-595.

21. Petroianu A. Pesquisa experimental. In Petroianu A (ed) Ética, Moral e Deontologia Médicas. Rio de Janeiro. Ganabara Koogan, SA 200, pp. 185-190.

Endereço para correspondência para: Prof. Andy Petroianu Av. Afonso Pena, 1626 - Apto 1901 30130-005 - Belo Horizonte-MG

Fax: (31) 3274-7744 